\title{
Reoperations on the total aortic arch in 119 patients: Short- and mid-term outcomes, focusing on composite adverse outcomes and survival analysis
}

\author{
Ourania Preventza, MD, ${ }^{\mathrm{a}, \mathrm{b}}$ Andrea Garcia, MD, ${ }^{\mathrm{b}}$ Denton A. Cooley, MD, ${ }^{\mathrm{a}}$ Alexandra Tuluca, MD, \\ Katherine H. Simpson, MS, ${ }^{\mathrm{c}}$ Faisal G. Bakaeen, MD, ${ }^{\mathrm{a}, \mathrm{b}, \mathrm{c}}$ Shuab Omer, MD, ${ }^{\mathrm{b}, \mathrm{c}}$ Lorraine Cornwell, MD, ${ }^{\mathrm{b}, \mathrm{c}}$ \\ Todd K. Rosengart, MD, ${ }^{\text {, c }}$ and Joseph S. Coselli, MD ${ }^{\mathrm{a}, \mathrm{b}}$
}

Objectives: To determine the preoperative and perioperative risk factors that significantly predict adverse outcomes after total arch replacement in patients with previous proximal aortic surgery and to analyze patient survival.

\begin{abstract}
Methods: We performed univariate analysis and logistic regression on data extracted from a prospectively maintained database for 119 patients who had undergone total arch operations during a 7.5-year period. All patients had undergone previous proximal aortic surgery. The adverse outcome was defined as a single composite endpoint comprising operative mortality, permanent neurologic deficit, and renal failure necessitating permanent hemodialysis.
\end{abstract}

\begin{abstract}
Results: The incidence of the composite endpoint was $13.5 \%$ (16 of 119 patients). The univariate predictors were preoperative pulmonary disease $(P=.010)$, cardiac ischemia time $(P=.032)$, and cardiopulmonary bypass (CPB) time $(P=.073)$. On multivariate analysis, the following were predictors of the composite endpoint: preoperative pulmonary disease $(P=.036)$, CPB time $(P=.039)$, concomitant coronary artery bypass $(P=.0057)$, previous aortic valve replacement $(P=.027)$, and previous thoracoabdominal aortic aneurysm surgery $(P=.057)$. Multivariate analysis showed that the CPB time predicted mortality $(P=.0044)$, and previous thoracoabdominal aortic aneurysm surgery predicted stroke $(P=.034)$. The overall survival was $85.3 \%$ during a median follow-up of 4.76 years (95\% confidence interval, $4.2-5.1$ ).
\end{abstract}

Conclusions: Aortic arch reoperations, although technically demanding, can produce acceptable results. Preoperative pulmonary disease, CPB time, and concomitant coronary artery bypass predicted an adverse outcome. The CPB time predicted mortality, and previous thoracoabdominal aortic surgery predicted stroke. (J Thorac Cardiovasc Surg 2014;148:2967-72)

Since Cooley and colleagues ${ }^{1}$ reported the first aortic arch replacement in 1955 , the strategies for brain protection have significantly advanced and surgical techniques have been refined, leading to improved perioperative rates of death and neurologic events. ${ }^{2,3}$ The application of hypothermia in aortic surgery, introduced by Griepp and colleagues, ${ }^{4}$ revolutionized cerebral protection and changed

\footnotetext{
From the Department of Cardiovascular Surgery, ${ }^{\text {a }}$ Texas Heart Institute, Houston, Tex; Division of Cardiothoracic Surgery, ${ }^{\mathrm{b}}$ Michael E. DeBakey Department of Surgery, Baylor College of Medicine, Houston, Tex; and Michael E. DeBakey Veterans Affairs Medical Center, ${ }^{\mathrm{c}}$ Houston, Tex.

Disclosures: Ourania Preventza reports consulting fees from Medtronic. Joseph S. Coselli reports consulting fees from Medtronic, Vascutek, and W. L. Gore, and grant support from Vascutek. All other authors have nothing to disclose with regard to commercial support.

Read at The American Association for Thoracic Surgery Aortic Symposium, New York, New York, April 24-25, 2014.

Received for publication April 22, 2014; revisions received June 27, 2014; accepted for publication June 28, 2014; available ahead of print Aug 21, 2014

Address for reprints: Ourania Preventza, MD, Division of Cardiothoracic Surgery, Michael E. DeBakey Department of Surgery, Baylor College of Medicine, BCM 390, One Baylor Plaza, Houston, TX 77030 (E-mail: opsmile01@aol.com). $0022-5223 / \$ 36.00$

Copyright (c) 2014 by The American Association for Thoracic Surgery http://dx.doi.org/10.1016/j.jtcvs.2014.06.095
}

surgeons' ability to perform complex aortic arch reconstruction. Other new techniques introduced during the past decade have included the use of antegrade cerebral perfusion by Kazui and colleagues ${ }^{5}$ for brain protection and the flexible alternative to the island technique by Spielvogel and colleagues. ${ }^{6}$ Despite these improvements, aortic arch surgery has remained challenging. Reoperation of the aortic arch, especially in patients with previous proximal aortic surgery, is one of the most complicated and technically demanding operations and requires a surgical team with expertise and experience. In the published data, the preoperative and intraoperative risk factors have been described in relatively large series of first-time operations of the aortic arch. In the present study, we identified the preoperative and intraoperative risk factors for patients undergoing extended aortic arch operations who had undergone previous proximal aortic surgery, evaluated the adverse outcomes in this group, and performed a survival analysis.

\section{METHODS}

From January 2005 to August 2013, 119 consecutive patients with previous proximal aortic surgery underwent reoperation for total aortic arch 


\section{Abbreviations and Acronyms \\ $\mathrm{ACP}=$ antegrade cerebral perfusion \\ $\mathrm{CABG}=$ coronary artery bypass grafting \\ $\mathrm{CPB}=$ cardiopulmonary bypass \\ $\mathrm{ET}=$ elephant trunk \\ LCCA $=$ left common carotid artery \\ TAAA $=$ thoracoabdominal aortic aneurysm}

replacement at our institution. The only patients excluded were those whose previous surgery was aortic valve replacement or coronary artery bypass grafting (CABG). The mean patient age was $55.4 \pm 13.7$ years, and most were men $(n=89,74.8 \%)$. The institutional review board at Baylor College of Medicine approved the present study. The data were collected and analyzed from a prospectively maintained clinical database. The patients' preoperative characteristics and demographic data are listed in Table 1, and the association with the composite endpoint is presented in Table 2.

\section{Study Design, Variables, and Adverse Outcome}

The medical records, surgical diagrams, and operative reports were reviewed to verify the collected data. The follow-up data were obtained from the clinic visits, hospital medical records, Social Security Death Index, and telephone interviews with the patient. The adverse outcome was defined as a single composite endpoint that included operative mortality, permanent neurologic deficit, and renal failure necessitating hemodialysis at discharge. A permanent neurologic deficit was defined as stroke (new brain injury evident either clinically or radiographically after the procedure) or paraplegia. Operative mortality was defined as death within 30 days postoperatively or before hospital discharge. Preoperative pulmonary disease was defined as a history of restrictive or obstructive (history of asthma, chronic bronchitis, or emphysema) lung disease. We have previously defined our intraoperative times. ${ }^{7}$ In brief, the cardiopulmonary bypass (CPB) time was defined as the period in which the patient was receiving cardiopulmonary support, not including the circulatory arrest time. The antegrade cerebral perfusion (ACP) time was defined as the period of circulatory arrest during which the patient was receiving ACP. The circulatory arrest time was defined as the ACP time plus the cerebral circulatory arrest time (period without ACP). The myocardial ischemia time was the interval from the initiation of the circulatory arrest time (no patients received crossclamping before circulatory arrest) until clamp removal and restoration of coronary flow. As a part of the preoperative workup, the patients had undergone computed tomography, with or without contrast, depending on their renal function, to assess the proximity of the cardiac structures to the sternum.

\section{Operative Technique}

All patients had had previous surgery in the ascending aorta and/or proximal arch or the total arch. Of the 119 patients, 16 (13.5\%) had undergone $>1$ previous sternotomy. The procedural details and concomitant procedures are listed in Table 3, and the association of the intraoperative variables and the composite endpoint is presented in Table 4.

For arterial inflow for CPB, we used the right axillary artery $(\mathrm{n}=107$, $89.9 \%)$, innominate artery $(n=7,5.9 \%)$, carotid artery $(n=4,3.4 \%)$, or femoral artery $(\mathrm{n}=1,0.8 \%)$. An $8-\mathrm{mm}$ Dacron graft was attached end-to-side with a running 6.0 Prolene suture to the right axillary artery, innominate artery, ${ }^{7}$ and/or right or left carotid artery after $1 \mathrm{mg} / \mathrm{kg}$ of heparin was given, and the graft was connected to the arterial line. After lysis of any adhesions, CPB was initiated, with cooling to a nasopharyngeal temperature of $21^{\circ} \mathrm{C}$ to $24^{\circ} \mathrm{C}$. During the entire procedure, we monitored the near-infrared spectroscopy signal. ${ }^{7}$ Of the 76 patients $(63.9 \%)$ who underwent total aortic arch replacement with the elephant trunk (ET) procedure, classic ET repair was used in 66 and frozen ET in 10. The aortic arch repair was performed using different reconstruction techniques (Tables 3 and 4). For the Y-graft aortic arch repair, we used a prefabricated or a self-made bifurcated (Y-graft, $\mathrm{n}=46$ ), trifurcated (double Y-graft, $n=28)$, and a single graft $(n=4$; Vascutek; Terumo, Renfrewshire, Scotland). The most common diameter of the main trunk of the Y-graft used was $12 \mathrm{~mm}$; that of the side branches was usually 8 or $10 \mathrm{~mm}$. The traditional island technique for arch repair was used in 20 patients $(16.8 \%)$, with reimplantation of 3 ( $\mathrm{n}=15$ patients) or reimplantation of $2(\mathrm{n}=5$ patients) vessels. A multibranch graft was used in 12 patients $(10.1 \%)$. Finally, a combination of the island technique and the Y-graft technique was used in 9 patients $(7.6 \%)$. We have previously described our technique using the Y-graft. ${ }^{2}$ In brief, during cooling, the brachiocephalic vessels were exposed, and the trifurcated graft was sutured first to the left subclavian artery and then to the left common carotid artery (LCCA). Occasionally, depending on the anatomy, a single 8- to $12-\mathrm{mm}$ graft was attached into the left subclavian artery, followed by attachment of the Y-graft to the LCCA. ${ }^{2}$ When using the bifurcated or trifurcated graft technique, the reconstruction always started distally with reconstruction of the subclavian artery first and then moved proximally toward reconstruction of the LCCA. When the targeted temperature had been achieved, circulatory arrest was initiated with ACP (flow $10 \mathrm{~mL} / \mathrm{kg} / \mathrm{min}$ ), which was adjusted according to the near-infrared spectroscopic findings. A decrease in the near-infrared spectroscopy regional cerebral oxygen saturation reading of $>10 \%$ from the patient's baseline measurement prompted us to increase the flow to $\geq 13$ to $15 \mathrm{~mL} / \mathrm{kg} / \mathrm{min}$. ACP can be administered by way of the branches of the Y-graft. The innominate artery is transected and anastomosed to the main trunk of the bi- or trifurcated graft. After completion of the anastomosis of the head vessels, a clamp was usually placed to the proximal aspect of the graft to direct the flow from the right axillary artery or the innominate artery to the other head vessels. If pathologic features extended beyond the left subclavian, we performed an ET repair using a skirted ET graft (Vascutek; Terumo). In patients in whom the frozen ET technique was used, the stent graft was delivered antegrade under direct vision and over a stiff Amplatz wire (Boston Scientific, Marlborough, Mass). ${ }^{8}$ With the Y-graft approach, unlike the island technique, the distal anastomosis to the arch, even when the ET technique is used, can be performed proximally at the level of the innominate or LCCA, reducing the risk to the recurrent laryngeal nerve and allowing for simplified access for hemostasis.

\section{Statistical Analysis}

For univariate analysis, to test for significant differences between the 2 groups, we used the Student $t$ test for continuous variables and the chi-square test, or Fisher's exact test when necessary, for categorical variables. Nominal logistic regression was performed. The following 19 preoperative and intraoperative variables were used for the multivariate logistic regression analysis: age, sex, $>1$ previous sternotomy, emergency, preoperative pulmonary disease, renal failure, preoperative stroke, preoperative $\mathrm{CABG}$ or percutaneous transluminal coronary angioplasty, previous surgery aortic root, previous transverse arch surgery, previous proximal arch surgery, previous aortic valve replacement surgery with concomitant proximal aortic surgery, previous thoracoabdominal aortic aneurysm (TAAA) surgery, circulatory arrest time, CPB time, cardiac ischemia time, concomitant $\mathrm{CABG}$, concomitant aortic root surgery, and concomitant aortic valve surgery. To determine what other variables were close to being predictors of the outcome measures, a sensitivity analysis was performed by changing the limits on the $P$ values required for a variable to both enter and stay in the model. When the entry value was set at $P=.3$ and the stay value was set at $P=.1$, different variables were shown to contribute to the outcomes.

Survival functions were estimated using the Kaplan-Meier method. The interval to death from the date of the surgery was computed for patients 
TABLE 1. Preoperative patient characteristics and demographics

\begin{tabular}{lc}
\hline \multicolumn{1}{c}{ Variable } & Value \\
\hline Age & $55.4 \pm 13.7$ \\
Male sex & $89(74.8)$ \\
Urgent/emergency surgery & $14(11.8)$ \\
$>1$ Previous sternotomy & $16(13.5)$ \\
Preoperative pulmonary disease & $47(39.5)$ \\
Preoperative renal insufficiency (creatinine $\geq 1.5 \mathrm{mg} / \mathrm{dL})$ & $24(20.2)$ \\
History of stroke & $16(13.5)$ \\
History of percutaneous balloon angioplasty & $5(4.2)$ \\
Previous surgery & \\
$\quad$ Ascending with or without proximal arch replacement & $112(94.1)$ \\
Aortic root replacement & $55(46.2)$ \\
Thoracoabdominal aortic replacement & $26(21.9)$ \\
Aortic valve replacement + proximal aortic surgery* & $17(14.3)$ \\
Coronary artery bypass graft + proximal aortic surgery* & $11(9.2)$ \\
Transverse arch replacement $\dagger$ & $7(5.9)$ \\
\hline Data presented as mean \pm standard deviation for continuous variables and $\mathrm{n}(\%)$ for \\
categorical variables. Preoperative pulmonary artery disease significant for mortality \\
only $(P=.0136)$, and previous thoracoabdominal aortic replacement significant for \\
stroke only $(P=.040)$ *Patients with previous aortic valve replacement or repair only \\
or CABG only were not included in our study. $\dagger$ Three of these patients had pseudoa- \\
neurysm at the island anastomosis site, 3 had infection of a previously placed arch \\
graft, and 1 had fusiform aneurysm after previous patch repair of the arch.
\end{tabular}

who had died before November 30, 2013. All statistical analyses were conducted using Statistical Analysis Systems, version 9.1 (SAS Institute, Inc, Cary, NC).

\section{RESULTS \\ Operative Mortality}

Of the 119 patients included in the present study, 10 $(8.4 \%)$ died in hospital, 2 of whom had undergone urgent surgery $(P=.33$; Table 5$)$. Of these 10 patients, 3 died of heart failure and had been unable to be weaned off ventricular assist support and extracorporeal membrane oxygenator; 3 died of multiorgan failure; 1 had had an extensive stroke; 1 died of a ruptured thoracoabdominal aneurysm while waiting for a stage II ET procedure; and 2 underwent unsuccessful cardiopulmonary resuscitation for hypotension and unresponsiveness on day 44 and 12. Univariate analysis showed that among the preoperative and intraoperative variables, preoperative pulmonary disease $(P=.0136), \mathrm{CPB}(P=.051)$, and cardiac ischemia time $(P=.0045)$ were significant predictors of mortality. In addition, concomitant CABG was marginally associated with mortality $(P=.063)$. In the multivariate logistic regression model, only CPB time remained a predictor of operative mortality $(P=.0044)$.

\section{Neurologic Events}

Seven patients $(5.9 \%)$ experienced a permanent stroke, and two $(1.7 \%)$ experienced paraplegia (Table 5). The cumulative permanent neurologic deficit was 7.6\%. On univariate analysis, preoperative TAAA surgery was associated with stroke $(P=.040)$. In the logistic regression analysis
TABLE 2. Preoperative variables and association with composite endpoint (operative mortality, permanent neurologic deficit, renal failure requiring hemodialysis at discharge)

\begin{tabular}{lccc}
\hline & \multicolumn{2}{c}{ Composite endpoint } & \\
\cline { 2 - 3 } \multicolumn{1}{c}{ Variable } & No & Yes & $\boldsymbol{P}$ \\
& $(\mathbf{n}=\mathbf{1 0 3})$ & $(\mathbf{n}=\mathbf{1 6})$ & value \\
\hline Male sex & $77(74.8)$ & $12(75.0)$ & 1.00 \\
Urgent/emergency surgery & $11(10.7)$ & $3(18.8)$ & .40 \\
$>1$ Previous sternotomy & $14(13.6)$ & $2(12.5)$ & 1.00 \\
Preoperative pulmonary disease & $36(35.0)$ & $11(68.8)$ & .010 \\
Preoperative renal insufficiency & $21(20.4)$ & $3(18.8)$ & 1.00 \\
$\quad$ creatinine $\geq 1.5$ mg/dL) & & & \\
History of stroke & $15(14.6)$ & $1(6.3)$ & .69 \\
History of percutaneous balloon & $5(4.9)$ & $0 .(0.0)$ & 1.00 \\
$\quad$ angioplasty & & & \\
Previous surgery & & & \\
$\quad$ Ascending with or without proximal & $97(94.2)$ & $15(93.8)$ & 1.00 \\
$\quad$ arch replacement & & & \\
Aortic root replacement & $50(48.5)$ & $5(31.3)$ & .20 \\
$\quad$ AVR or repair & $13(12.6)$ & $4(25.0)$ & .24 \\
$\quad$ Thoracoabdominal aortic replacement & $20(19.4)$ & $6(37.5)$ & .10 \\
AVR or repair + proximal aortic & $13(12.6)$ & $4(25.0)$ & .24 \\
$\quad$ surgery* & & & \\
Coronary artery bypass + & $8(7.8)$ & $3(18.8)$ & .17 \\
$\quad$ proximal aortic surgery* & & & \\
Transverse arch replacement or & $7(6.8)$ & $0(0.0)$ & .59
\end{tabular}
repair

Age (y) for composite $55.2 \pm 14.1 \quad 57.3 \pm 11.5 \quad .56$

Data presented as mean \pm standard deviation for continuous variables and $\mathrm{n}(\%)$ for categorical variables. Preoperative pulmonary artery disease significant for mortality only $(P=.0136)$, and previous thoracoabdominal aortic replacement significant for stroke only $(P=.040)$. AVR, Aortic valve replacement. *Patients with previous AVR or repair only or CABG only were not included in our study. $†$ Three of these patients had pseudoaneurysm at the island anastomosis site, 3 had infection of a previously placed arch graft, and 1 had fusiform aneurysm after previous patch repair of the arch.

model, previous TAAA surgery remained an independent risk factor for stroke $(P=.034)$.

\section{Composite Endpoint}

A total of 16 patients (13.5\%) had a composite endpoint comprising operative mortality, permanent neurologic deficit, and renal failure requiring hemodialysis at discharge (Table 5). Of these patients, 3 underwent emergency surgery $(P=.40)$. The association between the preoperative and intraoperative variables and the composite endpoint is presented in Tables 2-4. Multivariate logistic regression analysis (Table 6) showed that preoperative pulmonary disease and concomitant CABG were risk factors for the composite endpoint $(P=.020$ and $P=.0099$, respectively). An additional sensitivity analysis showed that CPB time, concomitant $\mathrm{CABG}$, preoperative pulmonary dysfunction, and previous aortic valve replacement were independent predictors of the composite endpoint $(P=.039$, $P=.0057, P=.036$, and $P=.027$, respectively). In addition, previous surgery for TAAA was associated with the composite endpoint $(P=.053)$. 
TABLE 3. Operative details and concomitant procedures $(n=119)$

\begin{tabular}{lc}
\hline \multicolumn{1}{c}{ Variable } & n (\%) or mean \pm SD \\
\hline Aortic arch repair & $119(100)$ \\
With elephant trunk & $76(63.9)$ \\
Arch reconstruction & \\
$\quad$ Y-graft & $46(38.7)$ \\
$\quad$ Trifurcated (double Y) graft & $28(23.5)$ \\
$\quad$ Single graft & $4(3.4)$ \\
$\quad$ Island anastomosis & $20(16.8)$ \\
$\quad$ Multibranch graft & $12(10.1)$ \\
$\quad$ Combination of island and Y-graft & $9(7.6)$ \\
Aortic root replacement & $15(12.6)$ \\
Aortic valve replacement & $14(11.8)$ \\
CABG & $12(10.1)$ \\
Mitral valve repair & $1(0.8)$ \\
Tricuspid valve repair & $1(0.8)$ \\
CBP time (min) & $153.0 \pm 66.4$ \\
Circulatory arrest time (min) & $73.0 \pm 34.6$ \\
Cardiac ischemia time (min) & $96.5 \pm 47.4$ \\
\hline
\end{tabular}

$S D$, Standard deviation; $C A B G$, coronary artery bypass grafting; $C P B$, cardiopulmonary bypass.

The other postoperative complications and the composite adverse outcome are listed in Table 5. Figure 1 shows the Kaplan-Meir survival curve for the follow-up period. The median follow-up period was 4.76 years $(95 \%$ confidence interval, 4.2-5.1). Survival was $91.5 \%$ at 1.6 years, $89.3 \%$ at 2.4 years, and $88.1 \%$ at 3 years.

\section{DISCUSSION}

Complex aortic arch repair, especially in the reoperative setting, is one of the most challenging cardiovascular operations today and carries a significant risk of mortality and stroke. For such patients, an important concern beyond surviving the surgery is whether they will be able to return to normal function or will have significant morbidity. In the present report, we analyzed adverse outcomes as a single composite endpoint in patients with prior proximal aortic

TABLE 4. Intraoperative variables and association with composite endpoint (operative mortality, neurologic deficit, renal failure requiring hemodialysis at discharge)

\begin{tabular}{lccc}
\hline & \multicolumn{2}{c}{ Composite endpoint } & \\
\cline { 2 - 3 } \multicolumn{1}{c}{ Variable } & No $(\mathbf{n}=\mathbf{1 0 3})$ & Yes $(\mathbf{n}=\mathbf{1 6})$ & $\boldsymbol{P}$ value \\
\hline Aortic root replacement & $13(12.6)$ & $2(12.5)$ & 1.00 \\
Aortic valve replacement & $11(10.7)$ & $3(18.8)$ & .40 \\
CABG & $7(6.8)$ & $5(31.3)$ & .010 \\
CPB time (min) & $146.6 \pm 58.3$ & $194.5 \pm 97.3$ & .073 \\
Circulatory arrest time (min) & $70.5 \pm 31.9$ & $89.1 \pm 46.5$ & .14 \\
Cardiac ischemia time (min) & $92.8 \pm 45.5$ & $120.1 \pm 53.9$ & .032 \\
\hline
\end{tabular}

Data presented as mean \pm standard deviation for continuous variables and $\mathrm{n}(\%)$ for categorical variables. CPB time significant for mortality only $(P=.051)$; cardiac ischemia time significant for mortality only $(P=.0045)$; and CABG almost significant for mortality $(P=.063)$. $C A B G$, Coronary artery bypass grafting; $C P B$, cardiopulmonary bypass.
TABLE 5. Short-term outcomes $(n=119)$

\begin{tabular}{lr}
\hline \multicolumn{1}{c}{ Outcome } & n (\%) \\
\hline Operative mortality* & $10(8.4)$ \\
Postoperative stroke & $7(5.9)$ \\
Paraplegia & $2(1.7)$ \\
Neurologic deficit (stroke or paraplegia)* & $9(7.6)$ \\
Permanent renal failure & $1(0.8)$ \\
Composite (mortality, neurologic deficit, permanent & $16(13.5)$ \\
$\quad$ renal failure) & \\
Postoperative tracheostomy & $21(17.7)$ \\
Reoperation for bleeding & $16(13.5)$ \\
Postoperative myocardial infarct & $3(2.5)$ \\
\hline Data presented as n $(\%)$ *Some of the patients had $>1$ complication. &
\end{tabular}

surgery who had undergone a total arch operation. In our series, the incidence of the composite endpoint was $13.5 \%$.

In a recent series of 145 total arch repairs, Leshnower and colleagues ${ }^{9}$ reported excellent results. The overall mortality was $9.7 \%$ (elective, $10.7 \%$ vs emergency, $4.3 \% ; P=\mathrm{NS}$ ), the incidence of permanent neurologic event was $2.8 \%$ (with no cases of paraplegia), the transient neurologic event rate was $5.6 \%$, and the overall incidence of renal failure requiring dialysis was $2.8 \% .^{9}$ Our composite endpoint $(13.6 \%)$ was greater, but our individual adverse event rates of $8.4 \%$ for mortality and $0.8 \%$ for renal failure requiring hemodialysis at discharge were lower. However, our stroke rate was greater than that reported by Leshnower and colleagues. ${ }^{9}$ In their study, 54 patients $(37.2 \%)$ required "redo" sternotomy and only 23 patients $(15.9 \%)$ had undergone previous proximal aortic surgery. ${ }^{9}$ In a large series of 1007 consecutive patients reported by the National Cerebral and Cardiovascular Center in Japan, the in-hospital death rate was $4.7 \%$, and the overall incidence of permanent stroke was $3.3 \% .{ }^{10}$ In that study, 764 patients had undergone total or partial arch surgery and only 38 $(3.8 \%)$ had undergone redo aortic surgery. Our greater mortality $(8.4 \%)$ and stroke $(5.9 \%)$ rates can be explained by previous proximal aortic surgery in all our patients and the more complex operations they required. In the Japanese study, multivariate analysis showed that chronic obstructive pulmonary disease was an independent risk factor for early mortality $(P=.041)$, as was concomitant CABG $(P<.001) .{ }^{10}$ In our study, univariate analysis supported the findings of the Japanese study, indicating that preoperative pulmonary disease and concomitant CABG were associated with mortality $(P=.010$ and $P=.010$, respectively). Also, in our logistic regression model, pulmonary dysfunction and concomitant CABG were independent predictors for the composite endpoint of operative mortality, permanent neurologic event, and renal failure requiring hemodialysis at discharge $(P=.036$ and $P=.0057$, respectively; Table 6). In a study of 423 patients who had undergone arch replacement at Kobe University Hospital, the overall mortality was $4.5 \%$ and that of permanent 
TABLE 6. Multivariate logistic regression analysis results for composite endpoint (operative mortality, permanent neurologic deficit, renal failure requiring hemodialysis at discharge), mortality, and postoperative stroke

\begin{tabular}{|c|c|c|c|}
\hline Variable & $P$ value & OR & $\begin{array}{l}95 \% \text { CI } \\
\text { for OR }\end{array}$ \\
\hline \multicolumn{4}{|l|}{ Composite endpoint } \\
\hline Preoperative pulmonary disease & .020 & 4.04 & $1.25-13.090$ \\
\hline CABG & .0099 & 6.13 & $1.55-24.28$ \\
\hline \multicolumn{4}{|l|}{ Additional sensitivity analysis } \\
\hline Elective & .069 & 0.19 & $0.032-1.14$ \\
\hline Preoperative pulmonary disease & .036 & 4.15 & $1.10-15.66$ \\
\hline Previous AVR or repair & .027 & 6.00 & $1.23-29.22$ \\
\hline $\begin{array}{l}\text { Previous surgery for TA pathologic } \\
\text { features }\end{array}$ & .057 & 3.67 & $0.96-14.10$ \\
\hline CPB time & .039 & 1.01 & $1.00-1.016$ \\
\hline CABG & .0057 & 9.14 & $1.90-43.85$ \\
\hline \multicolumn{4}{|l|}{ Mortality } \\
\hline CPB time & .0044 & 1.012 & $1.004-1.020$ \\
\hline \multicolumn{4}{|l|}{ Postoperative stroke } \\
\hline $\begin{array}{l}\text { Previous surgery for TA pathologic } \\
\text { features }\end{array}$ & .034 & 5.46 & $1.14-26.162$ \\
\hline
\end{tabular}

$O R$, Odds ratio; $C I$, confidence interval; $A V R$, aortic valve replacement; $T A$, thoracoabdominal; $C P B$, cardiopulmonary bypass; $C A B G$, coronary artery bypass grafting.

neurologic injury was $3.3 \% .{ }^{3}$ However, no information was given regarding previous sternotomy or aortic surgery. ${ }^{11}$ Zierer and colleagues ${ }^{11}$ conducted a large study of 1002 patients, $318(32 \%)$ of whom had undergone total arch replacement and $10 \%$ of the entire group had had previous cardiac operations. They reported that early mortality, permanent neurologic outcome, and renal failure necessitating hemofiltration was $5 \%, 3 \%$, and $4 \%$, respectively, in the total group, which included hemiarch and total arch procedures. ${ }^{11}$ Although their study was large, the overall number of patients who had previously undergone sternotomy was relatively small, they included all previous cardiac operations, and they did not provide separate data for the total arch group. Nevertheless, Zierer and colleagues ${ }^{11}$ found that a history of stroke was an independent predictor for permanent neurologic injury. However, our results did not support this finding. In our study, previous surgery for thoracoabdominal pathologic findings was an independent risk factor for permanent stroke $(P=.034)$ and was associated with the composite endpoint $(P=.057)$. This can be explained because the patients with previous thoracoabdominal surgery had more extensive disease and a greater incidence of atheromatous aorta. In a study of 636 consecutive patients who underwent partial or total aortic arch surgery (217 total arches), the investigators at the Leipzig Heart Center reported that overall early mortality was $11 \%$ and that of permanent neurologic injury was $11 \% .^{12}$ In their study, $15.7 \%$ of the entire group had had previous cardiac surgery; however, the data for the previous proximal aortic surgery were not given. ${ }^{12}$ Their multivariate analysis showed that total arch replacement was an independent predictor of mortality and stroke. In addition, the CPB time was an independent factor for early mortality. We also found that the CPB time was an independent predictor for early mortality $(P=.0044)$. Furthermore, the CPB time has been shown by others to be an independent risk factor for mortality. ${ }^{13,14}$ The CPB time was also associated with the composite endpoint $(P=.039)$ in our multivariate analysis. ${ }^{15}$ In an excellent study by Leonytev and colleagues ${ }^{15}$ at Mount Sinai, of 100 patients who underwent reoperative transverse arch procedures, mortality was greater during the first year after reoperation than that for

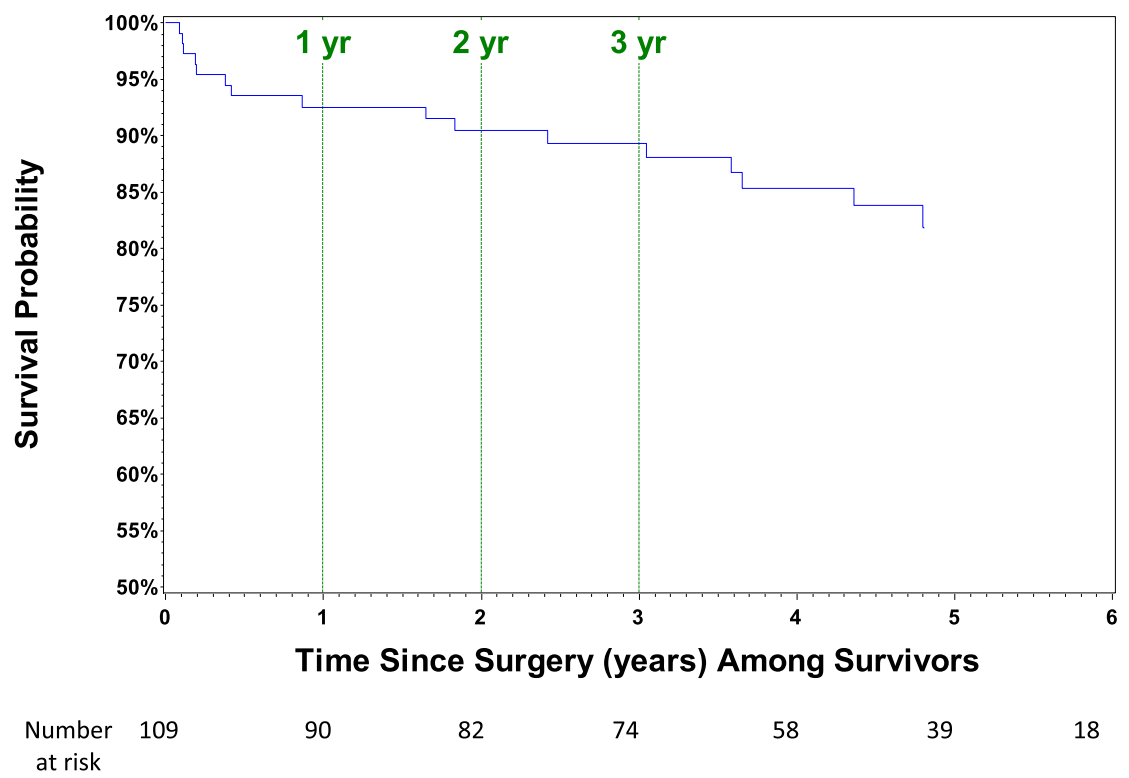

FIGURE 1. Kaplan-Meier survival curve for 109 surviving patients after hospital discharge, of whom 93 survived to the end of the study. 
patients undergoing primary arch surgery. However, the long-term survival did not differ between the patients who had undergone primary arch surgery and those who had undergone arch reoperation. ${ }^{15}$ They reported that chronic obstructive pulmonary disease was also an independent risk factor for mortality after arch replacement. ${ }^{15}$ Patel and colleagues ${ }^{14}$ conducted an analysis of 721 patients who had undergone extended arch repair during a 17-year period (23 of whom had had previous proximal aortic surgery, and 85 had had previous aortic valve or root surgery). They reported a 10 -year survival of $65 \%$. In our study, the median follow-up was 4.76 years, with an overall survival of $85.3 \%$. Higgins and colleagues ${ }^{16}$ studied the long-term outcomes after first-time thoracic aortic surgery in a population-based study of 1960 patients (330 arches, not redo) and reported that survival was $77.4 \%$ at 5 years for patients who underwent arch repair; their combined morbidity of mortality and stroke was $13.4 \%{ }^{16}$

In the cited studies from aortic centers of excellence, although the number of patients undergoing proximal arch and total arch replacement was large, the experience with patients undergoing reoperative total arch surgery with previous proximal aortic surgery was limited, except for the Hanover, Michigan, and Mount Sinai groups, which included 110, 108, and 100 patients, respectively, with combined previous cardiac and aortic reoperations. ${ }^{13-15}$

The main limitation of our study was its retrospective nature, with those inherent biases. Nevertheless, we believe that reporting the outcomes in this consecutive high-risk group by focusing on the adverse outcome of a composite endpoint is important.

To our knowledge, ours is the largest study in the published data of patients with previous proximal aortic operation who required total arch replacement with a focus on the composite endpoint of operative mortality, permanent neurologic deficit, and renal failure requiring hemodialysis at discharge. Our study has demonstrated that reoperation of the aortic arch after previous proximal aortic surgery, although technically challenging, is feasible with acceptable results.

\section{References}

1. Cooley DA, Mahaffey DE, DeBakey ME. Total excision of the aortic arch for aneurysm. Surg Gynecol Obstet. 1955;101:667-72.

2. LeMaire SA, Price MD, Parenti JL, Johnson ML, Lay AD, Preventza O, et al. Early outcomes after aortic arch replacement by using the Y-graft technique. Ann Thorac Surg. 2011;91:700-7; discussion 707-8.

3. Okita Y, Okada K, Omura A, Kano H, Minami H, Inoue T, et al. Total arch replacement using antegrade cerebral perfusion. J Thorac Cardiovasc Surg. 2013; 145:S63-71.

4. Griepp RB, Stinson EB, Hollingsworth JF, Buehler D. Prosthetic replacement of the aortic arch. J Thorac Cardiovasc Surg. 1975;70:1051-63.

5. Kazui T, Washiyama N, Muhammad BA, Terada H, Yamashita K, Takinami M, et al. Total arch replacement using aortic arch branched grafts with the aid of antegrade selective cerebral perfusion. Ann Thorac Surg. 2000;70:3-8; discussion 9.

6. Spielvogel D, Halstead JC, Meier M, Kadir I, Lansman SL, Shahani R, et al. Aortic arch replacement using a trifurcated graft: simple, versatile, and safe. Ann Thorac Surg. 2005;80:90-5; discussion 95.

7. Preventza O, Bakaeen FG, Stephens EH, Trocciola SM, de la Cruz KI, Coselli JS. Innominate artery cannulation: an alternative to femoral or axillary cannulation for arterial inflow in proximal aortic surgery. J Thorac Cardiovasc Surg. 2013; 145:S191-6.

8. Preventza O, Al-Najjar R, Lemaire SA, Weldon S, Coselli JS. Total arch replacement with frozen elephant trunk technique. Ann Cardiothorac Surg. 2013;2: 649-52.

9. Leshnower BG, Kilgo PD, Chen EP. Total arch replacement using moderate hypothermic circulatory arrest and unilateral selective antegrade cerebral perfusion. J Thorac Cardiovasc Surg. 2014;147:1488-92.

10. Iba Y, Minatoya K, Matsuda H, Sasaki H, Tanaka H, Kobayashi J, et al. Contemporary open aortic arch repair with selective cerebral perfusion in the era of endovascular aortic repair. J Thorac Cardiovasc Surg. 2013;145: S72-7.

11. Zierer A, El-Sayed Ahmad A, Papadopoulos N, Moritz A, Diegeler A, Urbanski PP. Selective antegrade cerebral perfusion and mild $\left(28^{\circ} \mathrm{C}-30^{\circ} \mathrm{C}\right)$ systemic hypothermic circulatory arrest for aortic arch replacement: results from 1002 patients. J Thorac Cardiovasc Surg. 2012;144:1042-9.

12. Misfeld M, Leontyev S, Borger MA, Gindensperger O, Lehmann S, Legare JF, et al. What is the best strategy for brain protection in patients undergoing aortic arch surgery? A single center experience of 636 patients. Ann Thorac Surg. 2012; 93:1502-8.

13. Khaladj N, Shrestha M, Meck S, Peterss S, Kamiya H, Kallenbach K, et al. Hypothermic circulatory arrest with selective antegrade cerebral perfusion in ascending aortic and aortic arch surgery: a risk factor analysis for adverse outcome in 501 patients. J Thorac Cardiovasc Surg. 2008;135:908-14.

14. Patel HJ, Nguyen C, Diener AC, Passow MC, Salata D, Deeb GM. Open arch reconstruction in the endovascular era: analysis of 721 patients over 17 years. J Thorac Cardiovasc Surg. 2011;141:1417-23.

15. Leontyev S, Misfeld M, Daviewala P, Borger MA, Etz CD, Belaev S, et al. Early- and medium-term results after aortic arch replacement with frozen elephant trunk techniques-a single center study. Ann Cardiothorac Surg. 2013;2:606-11.

16. Higgins J, Lee MK, Co C, Janusz MT. Long-term outcomes after thoracic aortic surgery: a population-based study. J Thorac Cardiovasc Surg. 2014; $148: 47-52$. 